ESJ Humanities

\title{
Déterminants de l'adoption des Variétés Améliorées de Maïs dans la Région de Sikasso Mali
}

\author{
Kone Bouréma \\ Dembele Bandiougou \\ Nientao Abdoulaye \\ Sidibe Moumoune \\ Kergna A. Oumar
}

Institut d'Economie Rurale/Programme ECOFIL, Mali

Doi:10.19044/esj.2021.v17n9p40

Submitted: 08 November 2020

Accepted: 15 February 2021

Published: 31 March 2021
Copyright 2021 Author(s)

Under Creative Commons BY-NC-ND

4.0 OPEN ACCESS

Cite As:

Bouréma K., Bandiougou D., Abdoulaye N., Moumoune S. \& Oumar K.A. (2021). Déterminants de l'adoption des Variétés Améliorées de Maïs dans la Région de Sikasso Mali. European Scientific Journal, ESJ, 17(9), 40. https://doi.org/10.19044/esj.2021.v17n9p40

\section{Résumé}

Le maïs à cause de sa forte productivité est parmi les céréales sèches qui occupent une place importante dans la stratégie de la sécurité Alimentaire au Mali. Vue cette place de ce produit dans l'économie des pays de l'Union Economique et Monétaire Ouest Africaine (UEMOA) en général et dans celle du Mali en particulier, le Conseil Ouest et Centre pour la Recherche et le Développement Agricoles (CORAF) a signé en 2014 avec ces pays, une convention pour la mise en œuvre d'un programme collaboratif sur la filière maïs. Il s'agit pour cette étude d'analyser les déterminants de l'adoption des variétés améliorées de maïs dans la région de Sikasso Mali. Ainsi, des données ont été collectées auprès de 200 producteurs de maïs dans deux cercles (Yanfolila et Koutiala) de la région de Sikasso. Les méthodes économétriques Probit, Logit avec l'approche contrefactuelle basée sur basée sur l'effet moyen de traitement (ATE) ont été utilisés. Il ressort des résultats que les facteurs déterminants dans l'adoption de la variété DEMBAYUNA sont le prix de vente et la participation à une formation. Par contre pour la variété SOTUBAKA, ce sont le nombre d'actif dans le ménage, l'appartenance à un groupement, le contact avec au moins un projet, le prix de vente et la participation à une formation qui sont déterminants. Le Taux commun d'adoption et d'exposition (JEA) est de 39\% pour la variété DEMBAYUMA 
et $64 \%$ pour la variété SOTUBAKA. L'amélioration des conditions des producteurs passe par une vulgarisation poussée des variétés performantes.

Mots-clés : Adoption, Probit, logit, Effet moyen de traitement (ATE), Maïs, Mali

\title{
Determinants of the Adoption of Improved Varieties of Maize in the Region of Sikasso Mali
}

\author{
Kone Bouréma \\ Dembele Bandiougou \\ Nientao Abdoulaye \\ Sidibe Moumoune \\ Kergna A. Oumar
}

Institut d'Economie Rurale/Programme ECOFIL, Mali

\begin{abstract}
Maize, due to its high productivity among dry cereals, occupies an important place in the food security strategy in Mali. Considering the importance of the product in the economy of West African Economics and Monetary Union (UEMOA) countries in general and in Mali in particular, the West and Central African Concil for Agricultural Research and Development (CORAF) in 2014 signed an agreement with these countries for the implementation of a collaborative program in the maize sector. This paper, therefore, focuses on assessing its impact on income, poverty, food and nutritional security, and on the education of children. Data were collected from 200 maize producers in two circles (Yanfolila and Koutiala) in the Sikasso region. The Probit and Logit econometric methods with the counterfactual approach based on the mean treatment effect (ATE) were used. The econometric results showed that the selling price and participation in training determined the adoption of DEMBAYUNA variety. The number of workers in the household, membership group, contact with at least one project, selling price, and participation in training influenced the uptake of SOTUBAKA variety. The Common Adoption and Exposure Rate (JEA) were 39\% for the DEMBAYUMA variety and $64 \%$ for the SOTUBAKA variety. The improvement of the livelihood of maize producers should be based on the strengthening of the extension services, i.e., high yielding varieties.
\end{abstract}

Keywords: Adoption, Mean Treatment Effect (ATE), Maize, Mali 


\section{Introduction}

Situé au cœur de l'Afrique de l'Ouest, le Mali est un pays sahélien enclavé d'une superficie totale de $1241238 \mathrm{Km}^{2}$. La population malienne est estimée à 18703435 habitants en 2017 et sera de 25584781 millions en 2025 suivant le taux d'accroissement intercensitaire $(3,6 \%)$ du Recensement Général de la Population et de l'Habitat de 2009. Les femmes prédominent légèrement (51\% de la population totale) et les ruraux représentent 77,6\% (EMOP, 2011) contre 73,2 \% en 2006. La population malienne, à majorité composée de jeunes, est concentrée dans la partie Sud du pays et le long du fleuve Niger (densité de 14 habitants au $\mathrm{Km}^{2}$ contre 8,87 habitants par $\mathrm{Km}^{2}$ de moyenne nationale.

L'économie a résisté cependant à ces différents chocs, et s'est redressée, affichant un taux de croissance de 1,7\% en 2013 et de 5,8\% en 2014. La contribution des activités agricoles au PIB demeure toujours élevée, avec $24 \%$ en moyenne sur la période 2006-2010 (INSTAT, 2014) environ 535 milliards de FCFA par an, hors les branches de la pêche, des produits forestiers et de cueillette. Les céréales sèches (mils, sorgho, maïs, fonio) ont contribué en moyenne pour 53\% dans la formation du PIB du secteur primaire sur la période 2006-2014.

Ce niveau de contribution est assuré par la production Agricole qui repose pour l'essentiel sur environ 800000 Exploitations Agricoles Familiales (EAF), d'une taille moyenne de 4,5 ha avec 9 à 10 personnes et dont $40 \%$ ont moins de 3 ha (RGA, 2005). Ces EAF intègrent, ou dans le meilleur des cas ont à leur aval, une multitude d'unités familiales de transformation agricole opérant pour la plupart à l'échelle artisanale, et quelques unités semi industrielles et industrielles (blé, riz, coton et canne à sucre).

Malgré tout, les agriculteurs sont les plus pauvres avec un taux de pauvreté de $57 \%, 81 \%$ pour l'incidence, $85 \%$ pour la profondeur et $86 \%$ pour la sévérité. L'extrême pauvreté (incapacité de répondre aux besoins nutritionnels de base) concernait $22 \%$ de la population en 2010. La baisse du taux de pauvreté nationale de 25,4 points constatée entre 1998 et 2010, (en moyenne $2,11 \%$ par an), est en train d'être rattrapée par l'accroissement général de la population $(3,6 \%)$.

Aussi, le niveau de développement humain du Mali reste très bas (176ème place - 0,407 en 2014-PNUD). La dynamique démographique (6,1 enfants par femme en âge de procréer) et le taux général de croissance démographique du pays traduisent l'importance des besoins à satisfaire au plan alimentaire et du bien-être social (éducation, santé .etc.).

Les mesures de politique agricole et commerciale décidées à compter du début de la décennie 90 ont eu pour conséquence l'accroissement de la production, aussi bien par la hausse des superficies que par l'augmentation des rendements moyens qui sont passés de $1000 \mathrm{~kg} / \mathrm{ha}$ à $2500 \mathrm{~kg} / \mathrm{ha}$ entre les 
années 1991 et 2010. L'État s'étant désengagé, l'écoulement sur le marché du maïs, qui est beaucoup moins demandé, est devenu plus incertain.

Compte tenu de sa productivité, aujourd'hui le maïs est au cœur de la stratégie de sécurité alimentaire au Mali.

Ainsi le CORAF et l'UEMOA ont signé en 2014 une convention pour la mise en œuvre d'un programme collaboratif pour l'amélioration des performances et de la compétitivité de la filière maïs.

L'accroissement de la productivité du maïs passe par l'accès et l'adoption des innovations technologiques développées par la recherche.

L'objectif de cette étude est d'analyser les déterminants de l'adoption de deux (2) variétés améliorées de maïs (Dembaniuma et Sotubaka).

\section{Matériel et méthode}

Pour mener à bien ce travail, des données ont été collecté auprès des producteurs de maïs dans les cercles de Koutiala et Yanfola.

\section{Enquête de terrain \\ 1.1. Echantillonnage}

\subsubsection{Choix des Zones}

Pour cette étude, l'enquête a été réalisée dans la Région de Sikasso qui produit $70 \%$ de la production nationale de maïs (CPS, 2015). A cet effet, les cercles retenus sont Koutiala et Yanfolila qui sont les plus grands bassins de production de maïs dans la région de Sikasso en dehors de la capitale régionale (Où les enquêtes en cette période sont très complexes). En plus, les deux cercles retenus sont différents du point de vue agro-écologique.

\subsubsection{Choix des Villages}

Les enquêtes ont été réalisées dans huit (8) villages (dans 4 communes) dont 4 villages par cercle. Ces villages sont retenus sur la base d'un choix raisonné pour tenir compte, de leur niveau de production de maïs et de la diversité des situations de chacune des zones. Ce choix a été fait en collaboration avec des personnes ressources ayant une bonne connaissance de ces deux Cercles (chefs secteurs agriculture).

Tableau I. Cercles, communes et villages retenus pour les enquêtes

\begin{tabular}{|l|l|l|}
\hline \multirow{2}{*}{ Cercle de Koutiala } & Communes & Villages \\
\cline { 2 - 3 } & Sinzina & Kaniko, Nampossela \\
\hline \multirow{2}{*}{ Cercle de Yanfolila } & Nampé & Ngolokouna, Baramba \\
\cline { 2 - 3 } & Gassoulou Balé, & Badogo, Ourou-Ourou \\
\cline { 2 - 3 } & Gouanan & Yorobougoula, Béréko \\
\hline
\end{tabular}




\subsubsection{Echantillon ménage}

Le nombre de ménage à enquêter par cercle a été calculé en faisant une pondération à partir de la production des deux cercles. Autrement dit, en tenant compte du poids (en pourcentage) de chaque cercle dans la production des deux zones

Tableau II. Répartition de l'échantillon par village

\begin{tabular}{|c|c|c|c|}
\hline \multirow{2}{*}{ Koutiala } & Commune & Koutiala & $\begin{array}{c}\text { Nombre de } \\
\text { ménage }\end{array}$ \\
\hline \multirow{3}{*}{ Sinsina } & Kaniko & 32 \\
\cline { 3 - 4 } & \multirow{2}{*}{ Nampé } & Nampossela & 32 \\
\cline { 3 - 4 } & & Ngolokouna, & 32 \\
\cline { 3 - 4 } & & Baramba & 32 \\
\hline \multirow{2}{*}{ Yanfolila } & \multirow{2}{*}{ Wassoulou } & Badogo, & $\mathbf{1 2 8}$ \\
\cline { 3 - 4 } & \multirow{2}{*}{ Balé, } & Ourou-Ourou & 18 \\
\cline { 3 - 4 } & \multirow{2}{*}{ Guana } & Yorobougoula, & 18 \\
\cline { 3 - 4 } & & Béréko & 18 \\
\hline \multicolumn{2}{|c|}{ S. Total } & & $\mathbf{7 2}$ \\
\hline \multicolumn{2}{|c|}{ TOTAL } & & $\mathbf{2 0 0}$ \\
\hline
\end{tabular}

Le choix des ménages a été réalisé de façon aléatoire à partir d'un pas de tirage calculé en fonction du nombre de producteur à enquêter. C'est-à-dire le nombre total d'exploitation/producteur du village a été divisé par le nombre à enquêter dans le village.

\subsubsection{Choix de variétés de maïs}

Pour cette étude deux variétés de maïs sont retenues, il s'agit de Dembanyuma et Sotubaka (Deux variétés crées par l'Institut d'Economie Rurale). Ceux deux variétés sont retenues à cause de leur importance relative dans la production dans notre zone études par suite d'enquêtes auprès des producteurs. Il ressort de notre enquête que parmi les variétés améliorées introduites par la recherche, ces deux variétés sont produites par $90 \%$ des producteurs de notre échantillon.

\subsection{Analyse des données}

Etant donné que l'étude a été réalisée dans les pays de l'UEMOA, une méthodologie commune a été adoptée au niveau des analyses. Après les enquêtes, les données ont été envoyées à la Coordination régionale pour traitement et les analyses ont été réalisées dans les différents pays.

Ainsi, les méthodes économétriques Probit, Logit avec l'approche contrefactuelle basée sur l'effet moyen de traitement (ATE) sont utilisés. 


\subsubsection{Modèle d'adoption des variétés améliorées de maïs}

Les taux d'adoption des technologies promues, ainsi que leurs déterminants ont été estimés par l'approche contrefactuelle basée sur l'effet moyen de traitement (ATE). L'approche contrefactuelle a été choisie pour corriger le biais d'exposition dont souffrent les méthodes économétriques Probit, Logit, Tobit qui sont couramment utilisées. La variable dépendante qui a été expliquée ici désigne l'adoption ou le rejet des technologies agricoles introduites. C'est une variable binaire prenant la valeur 1 lorsque le producteur a adopté et 0 en cas de non adoption. Les paramètres estimés au niveau de chaque technologie agricole sont :

ATE : le taux d'adoption potentiel. C'est la proportion des producteurs qui auraient adoptés les technologies agricoles s'ils étaient tous informés de leur existence ;

ATE1 : le taux d'adoption parmi les exposés c'est-à-dire la proportion de producteurs ayant adopté la technologie parmi ceux qui sont informés.

Les résultats de l'estimation varient selon l'approche utilisée. L'approche ATE paramétrique a été utilisée pour cette étude.

L'estimation paramétrique de l'ATE se base sur l'hypothèse d'indépendance conditionnelle et s'exprime de la manière suivante (Diagne \& Demont, 2007; Greene, 2002):

$$
\begin{aligned}
& \operatorname{ATE}(x)=E\left(\frac{Y_{i}}{x}\right)=E\left(\frac{Y}{x}, w=1\right) \\
& E\left(\frac{Y}{x}, w=1\right)=g(x, \beta)
\end{aligned}
$$

Avec :

$\mathrm{g}$ une fonction des vecteurs des covariantes $\mathrm{x}$;

$\beta$ un paramètre qui est estimé à partir du maximum de vraisemblance ; w le statut d'adoption.

1.2.2. Spécification et estimation des modèles : Les variables retenues pour cette étude sont

Age : c'est une variable continue qui est incluse dans le modèle d'adoption et les modèles d'impact (profit et sur dépenses totales du ménage). Plusieurs auteurs ont montré que les jeunes producteurs adoptent moins les technologies que les plus âgés (Bezu, et al., 2014; Ogada et al., 2014). Par contre, les résultats de Arodokoun (2011) et Glèlè et al. (2008) ont montré que les jeunes exploitants sont beaucoup plus disposés à adopter les innovations. L'âge peut donc avoir une influence positive ou négative sur l'adoption des variétés améliorées de maïs considéré dans le cas de cette étude comme technologie. 
L'appartenance à un groupement villageois de producteurs : c'est une variable binaire prenant les valeurs 1 si le producteur des variétés améliorées de maïs est membre d'un groupement de producteurs de maïs et 0 si non. Les producteurs appartenant à un groupement auront la tendance à avoir plus de connaissances/informations sur les nouvelles technologies agricoles innovantes à travers les échanges avec les autres producteurs. Plusieurs études ont montré un effet positif de l'appartenance à un groupement de producteurs sur l'adoption de technologies agricoles introduites (Mariano et al., 2012; Nigussie et al., 2017) (L'influence attendue de cette variable est positive).

L'expérience dans la production : c'est une variable continue qui désigne le nombre d'années d'expérience dans la production de maïs. Avec l'expérience, les producteurs peuvent devenir plus réticents ou plus ouverts aux innovations (CIMMYT, 1993 ; Mabah et al., 2013). Mais, selon Mabah et al. (2013), l'expérience devrait influencer négativement l'adoption des nouvelles technologies. On ne peut donc prévoit le signe de cette variable sur l'adoption des variétés améliorée.

Superficie totale emblavée : c'est une variable continue qui désigne la superficie totale de maïs cultivée. Notons que la superficie totale emblavée influence positivement, c'est-à-dire plus le producteur emblave et plus son capital est rentable (Biaou et al., 2016). Nous attendons donc un signe positif de cette variable sur le profit du producteur.

Nombre d'actifs dans le ménage : c'est une variable continue qui indique le nombre de membres du ménage travaillant avec le chef dans son exploitation agricole y compris le chef ménage. Le nombre d'actifs agricoles du ménage a été utilisé pour montrer son importance avec l'adoption d'une innovation (Ahouandjinou, 2008). On attend que cette variable ait une influence positive sur l'adoption des variétés améliorées de maïs.

Contact avec les agents de vulgarisation, de recherche et des projets/ONG : c'est une variable binaire qui prend la valeur 1 lorsque l'enquêté est en contact avec les agents de vulgarisation, de recherche et des projets/ONG et 0 si non.

Cette variable est considérée comme un élément indispensable dans l'adoption des nouvelles technologies (Manda et al., 2016; Kassie \& Tembo, 2016; Pratt \& Wingenbach, 2016; Wossen et al., 2017). Cette importance s'explique par le fait que c'est à travers le contact que le paysan accède à l'information sur l'existence d'une technologie nouvelle et les avantages y afférents. Le degré d'accès à l'information peut être déterminé par le contact avec les vulgarisateurs ou les chercheurs, par des démonstrations en milieu réel ou par des contacts avec les mass médias (Adegbola \& Arouna, 2003). Il est attendu un signe positif pour cette variable dans le modèle d'adoption. 


\section{Niveau Education Formelle}

C'est une variable polychotomique qui exprime les différents niveaux d'éducation dans l'enseignement général (primaire, secondaire et universitaire). Elle a été désagrégée en trois variables indicatrices dont deux, introduites dans les modèles. La troisième a été fixée. Chaque variable indicatrice prend la valeur 1 si l'enquêté a le niveau d'éducation recherché et 0 si non.

L'éducation formelle favorise l'adoption d'une nouvelle technologie (McBride \& El-Osta, 2002 ; Ouégraogo, 2003 ; Bravo-Ureta et al., 2005 ; Adégbola \& Adékambi, 2008). Il est prévu un signe positif pour cette variable.

\section{Participation à une formation dans le domaine agricole}

C'est une variable binaire qui prend la valeur 1 lorsque l'enquêté a participé à une formation dans le domaine agricole et 0 si non. Partant de l'idée que les rémunérations sont fonction de la productivité marginale, plus on se forme, plus le revenu que l'on tirera de cet investissement ultérieurement sera élevé (Becker, 1964).

\section{Résultats}

\section{Statistiques descriptives}

Les statistiques concernent les producteurs qui ont reçu l'information et qui ont adopté les variétés et les producteurs qui n'ont pas reçu l'information mais qui ont adopté les variétés et cela en tenant comptes des variables retenues dans notre modèle.

Tableau 1. Statistique descriptive des variables introduites dans le modèle d'adoption

\begin{tabular}{|l|l|l|}
\hline Variables & Information & Adoption \\
\hline Contact avec la vulgarisation (\%) & 23,62 & --- \\
\hline Distance du village de la ville ==<10 km & 7,54 & --- \\
\hline Education formelle (\%) & 11,62 & --- \\
\hline Appartenance à un groupement (\%) & 79,40 & 61,98 \\
\hline Contact avec les Projets /ONGs (\%) & 83,42 & 85,94 \\
\hline Nombre d'actifs dans le ménage & --- & $7,76(0,31)$ \\
\hline Participation à une formation agricole (\%) & --- & 53,65 \\
\hline Rendement meilleur des variétés améliorées (\%) & --- & 71,35 \\
\hline $\begin{array}{l}\text { Prix de vente meilleur pour les variétés améliorées } \\
(\%)\end{array}$ & --- & 46,88 \\
\hline Age révolu (ans) & --- & $\begin{array}{l}49,55 \\
(0,83)\end{array}$ \\
\hline
\end{tabular}

Source : données d'enquête, 2017

Le Tableau 1 renseigne la statistique descriptive des variables quantitatives et qualitatives des producteurs enquêtes. Cependant, les 
producteurs qui ont reçu l'information représentent $24 \%$ des producteurs qui sont en contact avec la vulgarisation agricole. Dans ce groupe, $8 \%$ sont situés à moins de $10 \mathrm{Km}$ de la zone urbaine et $12 \%$ ont reçu une éducation formelle. Le pourcentage des producteurs appartenant à un groupement recevant l'information est de $79 \%$ et $62 \%$ dans ce groupe ont adopté les variétés améliorées de maïs. Le pourcentage de producteurs (83\%) qui sont en contact avec les projets/ONG ont effectivement reçu l'information et $86 \%$ dans ce groupe ont adopté les variétés améliorées de maïs. Cela suppose que l'effet de groupe et le contact avec les $\mathrm{ONG} /$ projets favorisent l'accès à l'information et l'adoption des variétés améliorées.

Il existe des producteurs qui n'ont pas reçu l'information mais qui ont adopté les variétés améliorées de maïs. Les statistiques révèlent que $54 \%$ des adoptants ont participé à une formation agricole, $71 \%$ ont un rendement meilleur avec les variétés améliorées, $46 \%$ reçoivent un prix de vente meilleur pour les variétés améliorées et $49 \%$ de ces producteurs ont atteint l'âge révolu (49 ans).

\section{Déterminants d'adoption de variétés améliorées}

Le Tableau 2 nous informe sur l'effet de l'adoption des variétés améliorées 
Tableau 2. Résultats économétriques des facteurs affectant l'information et l'adoption des variétés

\begin{tabular}{|c|c|c|c|}
\hline Variables & $\begin{array}{l}\text { Au moins } \\
\text { une variété }\end{array}$ & DEMBANYUMA & SOTUBAKA \\
\hline \multicolumn{4}{|l|}{ Equation Information } \\
\hline Distance du village de la ville $==<10 \mathrm{~km}$ & $-0,33$ & $1,09 * *$ & $1,68 * * *$ \\
\hline Contact avec au moins un projet & $1,16 * * *$ & 0,45 & $1,12 * * *$ \\
\hline Education formelle & 0,77 & 0,01 & 0,91 \\
\hline Appartenance à un groupement & 0,28 & $0,53 * *$ & 0,34 \\
\hline Constante & 1,09 & $-0,45$ & 0,13 \\
\hline Nombre observations & 206 & 206 & 206 \\
\hline LR chi2 (df) & $15,54 * * *$ & $11,67 * *$ & $72,92 * * *$ \\
\hline Pseudo R2 & 0,22 & 0,04 & 0,35 \\
\hline Log likelihood & $-26,05$ & $-126,19$ & $-64,93$ \\
\hline Wald-LR chi2 (df) & 3 & 3 & 3 \\
\hline \multicolumn{4}{|l|}{ Equation Adoption } \\
\hline Nombre d'actif agricole dans le ménage & $-0,05$ & 0,007 & $0,08 * *$ \\
\hline Appartenance à un groupement & $0,78 *$ & 0,42 & $0,56^{*}$ \\
\hline Age & 0,01 & $-0,01$ & 0,00 \\
\hline Contact avec au moins un projet & $1,81 * * *$ & 0,57 & $0,85 *$ \\
\hline Rendement meilleur des variétés améliorées & 0,33 & 0,44 & 0,22 \\
\hline Prix de vente meilleur pour les variétés améliorées & 0,05 & $1,11 * * *$ & $1,26 * * *$ \\
\hline Participation à une formation & 0,38 & $1,00 * * *$ & $0,61 *$ \\
\hline Constante & 0,10 & $1,28 *$ & 0,47 \\
\hline Nombre observations & 198 & 136 & 166 \\
\hline LR chi2 (df) & $21,25 * * *$ & $35,11 * * *$ & $33,13 * * *$ \\
\hline Pseudo R2 & 0,35 & 0,19 & 0,20 \\
\hline Log likelihood & $-19,64$ & $-74,21$ & $-66,22$ \\
\hline Wald-LR chi2 (df) & 6 & 6 & 6 \\
\hline ATE & $0,95 * * *$ & $0,62 * * *$ & $0,78 * * *$ \\
\hline ATE1 & $0,96 * * *$ & $0,59 * * *$ & $0,79 * * *$ \\
\hline ATE0 & $0,82 * * *$ & $0,69 * * *$ & $0,72 * * *$ \\
\hline
\end{tabular}

Code $: * ; * ; * * *$. Significatif respectivement à $10 \% ; 5 \%$ et $1 \%$ Source : données enquête

Pour l'information, l'analyse statistique LR chi2 est significative à $1 \%$ et $5 \%$ et le Pseudo R2 est significative. Les facteurs qui ont contribué à l'information des producteurs sur la variété DEMBAYUMA sont la distance et l'appartenance à un groupement. Les facteurs qui ont contribué à l'information des producteurs sur la variété SOTUBAKA sont la distance et le contact avec un projet. Et le facteur qui a contribué à l'information des producteurs sur au moins une variété est le contact avec au moins projet.

Pour l'adoption, l'analyse statistique LR chi2 est significative à $1 \%$ et le Pseudo R2 est significative. Les facteurs déterminants dans l'adoption de la 
variété DEMBAYUNA sont le prix de vente et participation à une formation. Les facteurs déterminants dans l'adoption de la variété SOTUBAKA sont le nombre d'actif dans le ménage, l'appartenance à un groupement, contact avec au moins un projet, le prix de vente et la participation à une formation. Pour l'adoption d'au moins une variété, les facteurs déterminants sont, l'appartenance à un groupement et le contact avec un projet.

\section{Taux d'adoption des variétés améliorés}

Tableau 3. Taux d'adoption des deux variétés de maïs les plus adoptées

\begin{tabular}{|l|c|c|c|}
\hline Paramètres & $\begin{array}{c}\text { Au moins } \\
\text { une variété }\end{array}$ & DEMBANYUMA & SOTUBAKA \\
\hline Taux d'adoption potentiel (ATE) & $0,95 * * *$ & $0,62 * * *$ & $0,78^{* * *}$ \\
\hline Taux d'adoption parmi les exposés (ATE1) & $0,96 * * *$ & $0,59 * * *$ & $0,79 * * *$ \\
\hline Taux d'adoption parmi les non exposés (ATE0) & $0,82 * * *$ & $0,69 * * *$ & $0,72 * * *$ \\
\hline Taux Commun d'adoption et d'exposition (JEA) & $0,92 * * *$ & $0,39 * * *$ & $0,64 * * *$ \\
\hline Écart d'adoption (GAP=JEA-ATE) & $-0,032 * * *$ & $-0,23 * * *$ & $-0,14 * * *$ \\
\hline Biais de sélection de la population (PBS=ATE1- ATE) & $0,005^{* *}$ & $-0,03 * * *$ & 0,01 \\
\hline
\end{tabular}

Code : **; $* * *$. Significatif respectivement à $5 \%$ et $1 \%$

Source : données enquête

Par définition, le taux d'adoption potentiel (ATE) est la proportion des producteurs qui auraient adopté les variétés améliorées de maïs s'ils étaient tous informés. Le taux d'adoption potentiel d'au moins une variété améliorée est de $95 \%$. Le taux d'adoption potentiel de la variété DEMBANYUMA et de SOTUBAKA est respectivement, $62 \%$ et $78 \%$.

Parmi les producteurs qui ont reçu les informations sur les variétés améliorées de maïs dans la zone de Sikasso, $96 \%$ ont au moins adopté une variété, $59 \%$ ont adopté la variété DEMBAYUMA et 79\% ont adopté la variété SOTUBAKA. Par contre, les producteurs qui n'ont pas reçu les informations mais qui ont adopté les variétés sont $82 \%$ pour au moins une variété, $69 \%$ pour la variété DEMBANYUMA et $72 \%$ pour SOTUBAKA.

Le Taux commun d'adoption et d'exposition (JEA) qui signifie que la proportion des producteurs qui ont été informés sur les variétés améliorées et qui les ont adoptées sont de $92 \%$ pour au moins une variété, 39\% pour la variété DEMBAYUMA et $64 \%$ pour la variété SOTUBAKA. La différence entre JEA et ATE donne l'écart d'adoption. Donc les écarts d'adoption sont de $3 \%, 23 \%$ et $14 \%$, respectivement pour d'au moins une variété, la variété DEMBAYUMA et la variété SOTUBAKA. L'analyse statistique révèle qu'il y'a une différence significative entre les producteurs adoptants DEMBAYUMA et SOTUBAKA.

Le PBS est statistiquement significatif on rejette donc l'hypothèse nulle que la sous-population actuellement informée a probablement adopté les 
variétés améliorées de maïs comme l'aurait adopté toute la population. Donc les producteurs exposés n'ont pas la même chance d'adopter au moins une des variétés améliorées de maïs que les producteurs qui ne sont pas exposés.

\section{Discussion}

Cette étude met en évidence les facteurs qui déterminent l'adoption des variétés de maïs au Mali. Ces facteurs sont la distance, le contact avec au moins un projet, le nombre d'actif, le prix de vente. L'étude réalisée par Laure et al. (2013) sur l'adoption des technologie du maïs au Cameroun a montré que sur la probabilité d'adopter le paquet technique augmente chez les exploitants agricoles qui sont en contact avec des agents de vulgarisation et participe à des démonstrations organisées par ceux-ci a une probabilité d'adopter le paquet technique vulgarisé plus grande que ses pairs. En effet, être en contact avec des services de vulgarisation accroît de près de $2,4 \%$ la probabilité d'adopter le paquet technique.

Une autre étude réalisée sur le niébé par Adéoti et al. (2002) montre que les variables contact avec la vulgarisation, Revenu extra et la taille du ménage) influencent positivement et de façon significative l'adoption des variétés améliorées de niébé ?

\section{Conclusion}

Il ressort de cette étude que le maïs joue un rôle important dans l'économie des exploitations agricole au Mali.

Favorisée par le système coton à travers l'octroi des engrais, cette culture connait aujourd'hui une extension des superficies avec un certain niveau d'amélioration du rendement à l'hectare. Mais force est de reconnaitre que cette productivité est affectée souvent par la mauvaise pluviométrie car cette culture réagit fortement au stress hydrique.

Il serait donc nécessaire de :

- Créer des variétés performantes résistantes au stress hydrique ;

- Continuer la vulgarisation des variétés performantes.

\section{References:}

1. Adégbola, Y. P., Arouna, A. \& Adékambi, A. S. (2011). Impact de l'adoption des variétés améliorées de maïs et de niébé au Bénin. 68p.

2. Adéoti, R., Coulibaly, O., \& Temo, M. (2002). Facteurs affectant l'adoption des nouvelles technologies du niébé Vigna unguiculata en Afrique de l'Ouest Bulletin de la Recherche Agronomique du Bénin. Numéro 36 - Juin 2002, 18p.

3. Asfaw, S., Shiferaw, B., Simtowe, F., \& Lipper, L. (2012). Impact of modern agricultural technologies on smallholder welfare: Evidence from Tanzania and Ethiopia. Food policy, 37(3), 283-295. 
4. Bengaly, A.Z. (2015). Evaluation d'impact de l'adoption des technologies sur la production céréalière des ménages du sahel en Afrique de l'Ouest

5. Bezu, S., Kassie, G. T., Shiferaw, B., \& Ricker-Gilbert, J. (2014). Impact of improved maize adoption on welfare of farm households in Malawi: a panel data analysis. World Development, 59, 120-131.

6. Biaou, D., Yabi, J. A., Yegbemey, R. N., \& Biaou, G. (2016). Performances technique et économique des pratiques culturales de gestion et de conservation de la fertilité des sols en production maraîchère dans la commune de Malanville, Nord Bénin. International Journal of Innovation and Scientific Research, 21(1), 201-211.

7. CNS-Mais/ CORAF (2018). Impact de l'adoption des variétés améliorées de maïs sur le bien-être des maïsiculteurs au Benin, au Burkina-Faso, en Côte d'ivoire et au Mali. Rapport d'étude régionale, $59 \mathrm{p}$.

8. CSA/RNDSA (2016). Etude d'insertion du maïs dans le stock national de sécurité (sns), rapport d'étude, Bamako, 77p.

9. Dembélé, M., Gourichon, H., \& Koné, B., (2014). Analyse des incitations par les prix pour maïs au Mali. Série de notes techniques, SAPAA, FAO, Rome.

10. Diagne, A., Dontsop-Nguezet, P. M., Kinkingninhoun-Medgabé, F. M., Alia, D., Adegbola, P. Y., Coulibaly, M., ... \& Ojehomon, V. T. (2012, August). The impact of adoption of NERICA rice varieties in West Africa. In SPIA Pre-conference workshop (Vol. 28).

11. Foster, J., Greer, J., \& Thorbecke, E. (1984). A class of decomposable poverty measures. Econometrica: journal of the econometric society, 761-766.

12. Goubalan (2011). Revue documentaire sur les filières agricoles et le crédit vivrier au Bénin : Cas de la filière maïs, Rapport PAPA

13. Greene, W. H. (2002). Econometric analysis Seventh Edition.

14. Heckman, J. J. (2010). Building bridges between structural and program evaluation approaches to evaluating policy. Journal of Economic literature, 48(2), 356-98.

15. Heckman, J. J., \& Vytlacil, E. (2005). Structural equations, treatment effects, and econometric policy evaluation 1. Econometrica, 73(3), 669-738.

16. Heckman, J. J., \& Vytlacil, E. J. (1999). Local instrumental variables and latent variable models for identifying and bounding treatment effects. Proceedings of the national Academy of Sciences, 96(8), 47304734.

17. INSTAT (2014). Le Mali en Chiffre, Bamako. 
18. Manda, J., Alene, A. D., Gardebroek, C., Kassie, M., \& Tembo, G. (2016). Adoption and impacts of sustainable agricultural practices on maize yields and incomes: Evidence from rural Zambia. Journal of Agricultural Economics, 67(1), 130-153.

19. Mariano, M. J., Villano, R., \& Fleming, E. (2012). Factors influencing farmers' adoption of modern rice technologies and good management practices in the Philippines. Agricultural Systems, 110, 41-53.

20. Nigussie, Z., Tsunekawa, A., Haregeweyn, N., \& Adgo, E. (2017). Factors influencing small-scale farmers ' adoption of sustainable land management technologies in north-western Ethiopia. Land Use Policy, 67(May), 57-64.

21. Ogada, M. J., Mwabu, G., \& Muchai, D. (2014). Farm technology adoption in Kenya: a simultaneous estimation of inorganic fertilizer and improved maize variety adoption decisions. Agricultural and food economics, 2(1), 12.

22. Pratt, O. J., \& Wingenbach, G. (2016). Factors affecting adoption of green manure and cover crop technologies among Paraguayan smallholder farmers. Agroecology and Sustainable Food Systems, 40(10), 1043-1057.

23. Tene, M., Laure, G., Temple, L., \& Havard, M. (2013). Les déterminants de l'adoption d'innovations techniques sur maïs à l'ouest Cameroun, une contribution à la sécurisation alimentaire. PAG.

24. Wossen, T., Abdoulaye, T., Alene, A., Haile, M. G., Feleke, S., Olanrewaju, A., \& Manyong, V. (2017). Impacts of extension access and cooperative membership on technology adoption and household welfare. Journal of rural studies, 54, 223-233. 